\title{
INTRODUCTION TO THIRD-ORDER JACOBSTHAL AND MODIFIED THIRD-ORDER JACOBSTHAL HYBRINOMIALS
}

\author{
Gamaliel Cerda-Morales \\ Instituto de Matemáticas \\ Pontificia Universidad Católica de Valparaíso \\ Blanco Viel 596, Cerro Barón, Valparaíso, Chile \\ e-mail: gamaliel.cerda.m@mail.pucv.cl
}

\begin{abstract}
The hybrid numbers are generalization of complex, hyperbolic and dual numbers. In this paper, we introduce and study the third-order Jacobsthal and modified third-order Jacobsthal hybrinomials, i.e., polynomials, which are a generalization of the Jacobsthal hybrid numbers and the JacobsthalLucas hybrid numbers, respectively.

Keywords: third-order Jacobsthal numbers, recurrence relations, complex numbers, hyperbolic numbers, dual numbers, polynomials.
\end{abstract}

2010 Mathematics Subject Classification: 11B37, 11B39.

\section{REFERENCES}

[1] G. Cerda-Morales, Identities for third order Jacobsthal quaternions, Adv. Appl. Clifford Alg. 27 (2017) 1043-1053.

doi:10.1007/s00006-016-0654-1

[2] G. Cerda-Morales, On a generalization of Tribonacci quaternions, Mediterranean J. Math. 14 (2017) 1-12.

doi:10.1007/s00009-017-1042-3

[3] G. Cerda-Morales, A note on modified third-order Jacobsthal numbers, Proyecciones J. Math. 39 (2020) 731-747.

doi:10.22199/issn.0717-6279-2020-02-0025

[4] G. Cerda-Morales, Some results on dual third-order Jacobsthal quaternions, FILOMAT 33 (2019) 1865-1876.

doi:10.2298/FIL1907865C 
[5] G. Cerda-Morales, Third-order Jacobsthal generalized quaternions, J. Geom. Symmetry Phys. 50 (2018) 11-27.

doi:10.7546/jgsp-50-2018-11-27

[6] C.K. Cook and M.R. Bacon, Some identities for Jacobsthal and Jacobsthal-Lucas numbers satisfying higher order recurrence relations, Ann. Math. Inform. 41 (2013) $27-39$.

[7] G.B. Djordjević, Generalized Jacobsthal polynomials, Fibonacci Quart. 38 (2000) 239-243.

[8] G.B. Djordjević and H.M. Srivastava, Incomplete generalized Jacobsthal and Jacobsthal-Lucas numbers, Math. and Comp. Modelling 42 (2005) 1049-1056. doi:10.1016/j.mcm.2004.10.026

[9] A.F. Horadam, Jacobsthal representation polynomials, Fibonacci Quart. 35 (1997) 137-148.

[10] A.F. Horadam, Jacobsthal representation numbers, The Fibonacci Quarterly 43 (1996) 40-54.

[11] M. Özdemir, Introduction to hybrid numbers, Adv. Appl. Clifford Alg. 28 (2018). doi:10.1007/s00006-018-0833-3

[12] A. Szynal-Liana, The Horadam hybrid numbers, Discuss. Math. Gen. Alg. Appl. 38 (2018) 91-98. doi:10.7151/dmgaa.1287

[13] A. Szynal-Liana and I. Włoch, The Fibonacci hybrid numbers, Util. Math. 110 (2019) 3-10.

[14] A. Szynal-Liana and I. Włoch, On Jacobsthal and Jacobsthal-Lucas Hybrid Numbers, Ann. Math. Silesianae 33 (2019) 276-283. doi:10.2478/amsil-2018-0009

[15] A. Szynal-Liana and I. Włoch, Introduction to Fibonacci and Lucas hybrinomials, Complex Variables and Elliptic Equations (2019).

doi:10.1080/17476933.2019.1681416

Received 20 March 2020

Revised 22 June 2020

Accepted 23 November 2020 